\title{
Differences in the Diversity and Structure of the Gut Microbiome in Different Life Stages of the American Cockroach (Periplaneta americana)
}

\section{Zhiyu Chen ( $\nabla$ zy.chen2@siat.ac.cn )}

Guangdong Pharmaceutical University https://orcid.org/0000-0002-0637-4914

\section{Bin Yang}

Guangdong Pharmaceutical University

\section{Peiyu Ou}

Guangdong Pharmaceutical University

\section{Xiaobao Jin}

Guangdong Pharmaceutical University

\section{Research article}

Keywords: Gut microbiome, Periplaneta americana, 16S rRNA high-through genes sequencing, Management strategies

Posted Date: March 11th, 2020

DOl: https://doi.org/10.21203/rs.3.rs-16743/v1

License: (c) (i) This work is licensed under a Creative Commons Attribution 4.0 International License. Read Full License 


\section{Abstract}

Gut microbes play critical roles in host nutrition, physiology, and behavior. Periplaneta americana is a famous urban pest which is widely distributed in the tropics and subtropics, but very few information is available on the gut microbiome of Periplaneta americana, particularly in its different life stages. Here, we characterized the diversity and structure of gut microbiome in eggs, nymph and adult life stages of Periplaneta americana using high-throughput 16S rRNA genes sequencing. Both the results of Alpha- and Beta-diversity analysis showed the diversity and structure of gut microbiome were significant different among the eggs, nymph and adult stages. The result of species distribution showed the predominant phyla in three life stages were Bacteroidetes, Firmicutes and Proteobacteria , but the relative abundances of these bacteria were significant different among each life stage. 1,169 operational taxonomic units were shared by three stages, which indicating the gut microbiome may be inherited to offspring from parents of Periplaneta americana. According to the prediction of functional genes in metabolic pathways, most of them were distributed in the metabolic pathways of basic physiology such as nutrition, growth, development and immunity, etc. The relative abundances of functional genes in metabolic pathways were significant different among life stages of Periplaneta americana, indicating the gut microbiome might play an important role in the physiology across its different life stages. This study revealed the diversity and structure of gut microbiome in different life stages of Periplaneta americana, which may contribute to us to understand it's physiology and behaviors.

\section{Introduction}

The American cockroach, Periplaneta americana, is one of the most diverse and abundant pests in the world (Nasirian 2018; Li et al 2018). Similar to other hemimetabolous insects, its life cycle can be divided into egg, nymph, and adult stages (Mullins 2015; Bell and Adiyodi 1981). Periplaneta americana can survive in a wide variety of habitats due to its strong environmental adaptability, and it prefers moist, shady, and warm areas so that it is famous for its household name "Waterbug". Periplaneta americana is also a serious urban pest due to its ability to act as a carrier and transfer many disease-causing organisms, such as bacteria, viruses and parasites (Baggio-Deibler et al 2018; Yun et al 2017; Pai 2015). Periplaneta americana can become a potential model to study the biology of a hemimetabolous pests due to their rapid growth, high fecundity, and remarkable environmental adaptability.

Similar to other animals, the gut microbiome is one of the most important divisions of the insect gut system, contributing to host fitness, particularly to nutritional needs, immunological functions, and defense against pathogens (Douglas 2014; Engel and Moran 2013a; Kwong et al 2017; Kaltenpoth et al 2014). In recent years, several studies have revealed that the effects of the gut microbiome also extend to systemic physiology, such as the immunity, metabolism, development and behaviors of the host (Shin et al 2011; Engel and Moran 2013b; Crotti et al 2012). Thus, the gut microbiome can be integrated into the biological system of the host and as a bacterial organ of the host (Bäckhed et al 2005), and it plays an important role in the overall health of its host. 
However, little is known about the gut microbiome of Periplaneta americana or other hemimetabolous insects, especially the characteristics of the gut microbiome in different life stages is still lacking. Ayako et al found that the gut microbiome of cockroaches is responsible for producing pheromones such as fatty acids, which could promote the social behaviors of cockroaches (Ayako et al 2015). Moreover, Tinker et al has revealed that Periplaneta americana hosts an abundant gut microbiome and has evolved unique mechanisms for maintaining stability of its gut microbiome (Tinker and Ottesen 2016). Therefore, the gut microbiome may be a curial factor which can strongly influence various physiological functions of Periplaneta americana. In this study, we characterized the structure and diversity of gut microbiome among eggs, nymph and adult life stages of Periplaneta americana, and revealed the relationship between the gut microbiome and the development of Periplaneta americana. Our results assisted to further understanding of the growth and development of Periplaneta americana, which may help in the development of effective management strategies of Periplaneta americana or other cockroaches.

\section{Materials And Methods}

Sample collection and DNA extraction

The Periplaneta americana insects in this study were reared and provided by Guangdong Provincial Center for Disease Control and Prevention. The cockroach adults and nymph were fed with dried wheat bran and water, and no feeding to eggs. The insects were paralyzed at $4{ }^{\circ} \mathrm{C}$ and washed alternately with water and $75 \%$ ethanol 3 times before dissection. The intestinal tracts were then quickly removed and washed carefully in sterile $1 \times$ PBS (Phosphate-buffered Saline). Samples were stored at $-80{ }^{\circ} \mathrm{C}$ until DNA extraction.

The frozen tissues were transferred to a sterile mortar into liquid N2 and ground with a sterile pestle. The DNA extraction of the samples, as well as the soil and root samples, were carried out using the PowerSoil DNA Isolation Kit (ANBIOSCI TECH LTD, Shenzhen, China), using instructions provided by the manufacturer.

PCR amplification, library preparation and high-throughput sequencing

The V3-V4 region of the bacterial 16S rRNA gene was amplified by PCR using the 338F/806R primers set (Yu et al 2005; Walters et al 2016) (338F: 5'-ACTCCTACGGGAGGCAGCA-3', 806R: 5'-

GGACTACHVGGGTWTCTAAT-3'). The PCR was performed in a total volume of $50 \mu \mathrm{L}$ containing $0.2 \mu \mathrm{L}$ Q 5 High-Fidelity DNA Polymerase, $10 \mu \mathrm{L}$ High GC Enhancer, $10 \mu \mathrm{L}$ Buffer, $1 \mu \mathrm{L}$ dNTP, $1.5 \mu \mathrm{L}$ of each primer $(10 \mu \mathrm{M}), 40-60 \mathrm{ng}$ of DNA template and deionized ultrapure water to $50 \mu \mathrm{L}$. The conditions of the PCR were as follows: denaturation step at $95^{\circ} \mathrm{C}$ for $5 \mathrm{~min} ; 15$ cycles at $95^{\circ} \mathrm{C}$ for $1 \mathrm{~min}, 50^{\circ} \mathrm{C}$ for $1 \mathrm{~min}$, and 72 ${ }^{\circ} \mathrm{C}$ for $1 \mathrm{~min}$; and a final extension at $72{ }^{\circ} \mathrm{C}$ for $7 \mathrm{~min}$. The PCR products were verified by $1.8 \%$ agarose gel electrophoresis and the samples with a bright main band of approximately $450 \mathrm{bp}$ were mixed in equidensity ratios. Then, the mixture of PCR products was purified by GeneJETGel Extraction Kit (Thermo Fisher Scientific, Waltham, MA, United States) and quantified with Qubit 2.0 Fluorometer (Thermo Fisher). The resultant products were sent for sequencing using the Illumina HiSeq 2500 platform (Illumina, 
Inc., San Diego, CA, United States) and the 16S rRNA libraries were generated at Biomarker Bioinformatics Technology, Co., Ltd. (Beijing, China).

Bioinformatic and statistical analysis

The overlapping regions between the paired-end were merged into longer single sequences using FLASH (v1.2.7) (Magoč and Salzberg 2011), and the raw reads were quality filtered under the specific filtering conditions (Bokulich et al 2013) to obtain high-quality clean tags on the basis of the quality-control program suite QIIME (v1.8.0) (Caporase et al 2010). Sequences that were less than $200 \mathrm{bp}$ in length or that contained homopolymers longer than $8 \mathrm{bp}$ were removed. The chimeric sequences were detected by comparing tags with the references database (RDP Gold database) and removed using the UCHIME algorithm. The effective sequences were obtained and used in the final analysis.

Operational taxonomic units (OTUs) were clustered on the basis of $97 \%$ similarity sequence identity using the clustering program UCLUST (v1.2.22) (Edgar 2010). Then OTUs were taxonomically classified to different levels of phylum, class, order, family, genus, and species by the Ribosomal Database Program (RDP) classifier.

Alpha-diversity analysis (i.e., Observed species, ACE, Chao 1, Shannon and Simpson indices) were calculated by QIIME (v.1.8.0) using for richness and diversity indices of the gut bacterial community in each sample. Beta-diversity analysis was calculated by unweighted UniFrac and non-metric multidimensional scaling (NMDS). A one-way analysis of similarity (ANOSIM) was performed to determine the differences in gut microbiome structures among eggs, nymph and adult stages. Linear discriminant analysis (LDA) effect size (LefSe) analysis was performed to reveal the significant differences in ranking of abundant among eggs, nymph and adult stages (Segata et al 2011). The functional biomarkers were discriminated using a size-effect threshold of 4.0 on the logarithmic LDA score. The functional gene content in the gut microbiome was predicted by Phylogenetic Investigation of Communities by Reconstruction of Unobserved States (PICRUst).

Alpha-diversity indices are presented as the means \pm SD. The differences in Alpha-diversity indices and relative abundances between groups at the phyla and genus levels were calculated by use of the Independent-sample $t$-test. The $p$-value $<0.05$ was considered the differences were statistically significant. The raw sequences obtained in this study have submitted to the NCBI Sequence Read Archive database under the SRA accession number PRJNA517316 (BioSample accession number SAMN10826798 to SAMN10826812).

\section{Results}

Analysis of $16 \mathrm{~S}$ rRNA Sequencing data

After pyrosequencing, quality filtering, and the removal of chimeric sequences, a total of $3,584,855$ clean tags and 173,805 - 220,205 (Mean 238,758 $\pm 33,191$ ) effective tags (Mean length $=422 \mathrm{bp}$ ) from per 
sample. At a sequence-similarity level of $97 \%$, the reads for the eggs, nymph and adult samples were assigned to 899, 1,422 and 1,428 OTUs (Table 1). The observed species (OTUs) in eggs, nymph and adult samples are shown in Fig. 2a.

Table 1

Richness and diversity estimates of gut microbiome in eggs, nymph and adult stages of Periplaneta americana

\begin{tabular}{|c|c|c|c|c|c|c|}
\hline Sample & $\begin{array}{l}\text { Tag } \\
\text { number }\end{array}$ & $\begin{array}{l}\text { OTU } \\
\text { numberb }^{b}\end{array}$ & Ace & Chao 1 & Simpson & Shannon \\
\hline Eggs & 971,740 & 899 & $\begin{array}{l}836.02 \pm \\
54.12\end{array}$ & $\begin{array}{l}842.03 \pm \\
54.96\end{array}$ & $\begin{array}{l}0.78 \pm \\
0.06\end{array}$ & $\begin{array}{l}0.84 \pm \\
0.18\end{array}$ \\
\hline Nymph & $1,305,334$ & 1,422 & $\begin{array}{l}1,131.89 \pm \\
26.53\end{array}$ & $\begin{array}{l}1,351.57 \pm \\
31.13\end{array}$ & $\begin{array}{l}0.01 \pm \\
0.002\end{array}$ & $\begin{array}{l}5.61 \pm \\
0.07\end{array}$ \\
\hline Adult & $1,304,299$ & 1,428 & $\begin{array}{l}1,198.15 \pm \\
23.18\end{array}$ & $\begin{array}{l}1,208.99 \pm \\
22.91\end{array}$ & $\begin{array}{l}0.05 \pm \\
0.02\end{array}$ & $\begin{array}{l}4.88 \pm \\
0.21\end{array}$ \\
\hline \multicolumn{7}{|c|}{$\begin{array}{l}\text { The table showing the numbers of Tag and OTUs in eggs, nymph and adult samples of Periplaneta } \\
\text { americana. The species richness and diversity were estimated with abundance-based coverage } \\
\text { estimator (Ace), Chao1, Shannon and Simpson indices. Each life stage included five biological } \\
\text { replicates. }\end{array}$} \\
\hline \multicolumn{7}{|c|}{$\begin{array}{l}\text { a Tag number after quality filtering and removal of chimeric sequences; } \\
\text { b Operational taxonomic units (OTUs) were defined by pairwise } 97 \% \text { sequence identity }\end{array}$} \\
\hline
\end{tabular}

The rarefaction curves for the OTUs indicated that the quantity of observed species increased as the sequencing depth increased at the beginning, and decreased while the rarefaction curves tapered off at the end. The rarefaction curves tended to approach the saturation plateau revealed that the quantity of sequencing in this study was adequate. (Fig. 2b). The Good's Coverage can reflect the database coverage rate of each sample, and its value in this study was close to $99 \%$, indicating that all samples reached sufficient sampling depth.

Differences of the gut microbiome diversity among the eggs, nymph and adult of Periplaneta americana

The richness and diversity of the gut microbial communities from all samples were assessed using the Alpha-diversity indexes, including the Ace, Chao 1, Simpson and Shannon estimator (Fig. 3). The Ace $(836.02 \pm 54.12,1,131.89 \pm 26.53,1,198.15 \pm 23.18)$, Chao $1(842.03 \pm 54.96,1,351.57 \pm 31.13,1,208.99 \pm$ 22.91), Simpson $(0.78 \pm 0.06,0.01 \pm 0.002,0.05 \pm 0.02)$ and Shannon $(0.84 \pm 0.18,5.61 \pm 0.07,4.88 \pm$ $0.21)$ indices were significant different among the eggs, nymph and adult of Periplaneta americana $(p<$ 0.05).

The difference in bacterial communities between groups were reflected by the Beta-diversity analysis. The Venn diagram of the eggs, nymph and adult samples showed that 1,169 OTUs were shared by the three groups and only a few OTUs (ranging 8-31) were unique present in one life stage of Periplaneta 
americana gut sample (Fig. 4). The NMDS plot showed that the microbial communities of eggs samples were different from the other groups, and it also revealed the distinct structure among the eggs, nymph and adult of Periplaneta americana (Fig. 5).

Relative Abundance and Core Gut Microbiome of the eggs, nymph and adult of Periplaneta americana

The top 10 representative phyla and genera according to relative abundance of the gut bacteria in eggs, nymph and adult stages that were displayed in Fig. 5. Bacteroidetes, Firmicutes and Proteobacteria were the predominant bacteria in every life stages at the phylum level, followed by Tenericutes and Fusobacteria (Fig. 6a). These bacteria accounted for $99.48,91.82$ and $96.65 \%$ of the detectable reads in the eggs, nymph and adult samples. The results of statistical analysis showed that the phyla in relative abundance with significant differences among eggs, nymph and adult stages were Bacteroidetes, Firmicutes and Proteobacteria $(p<0.05)$. Tenericutes showed significant differences between eggs and nymph $(p<0.05)$, and Fusobacteria showed significant differences between eggs and adult $(p<0.05)$. On genus level, Blattabacterium was the predominant genera in the eggs and adult samples, but it was hardly detected in the nymph samples. Desulfovibrio and Parabacteroides were the predominant genera in the nymph and adult samples (Fig. 6b). In the top 10 genera in relative abundance, Blattabacterium, Parabacteroides, Christensenellaceae, and Ruminococcaceae showed significant defferences among the eggs, nymph and adult stages $(p<0.05)$.

The core gut microbiomes of eggs, nymph and adult life stage were represented by the shared taxa within each group. The number of the shared OTUs was 334, 980 and 688 in the eggs (Fig. 7a), nymph (Fig. 7b) and adult (Fig. 7c) stages, respectively.

Significant Difference Analysis and Functional Genes Prediction among the eggs, nymph and adult of Periplaneta americana

The significant ranking of abundant modules and the different taxa among eggs, nymph and adult stages were revealed by LefSe analysis (Fig. 8a). The LDA scores displayed the intestinal microbial 47 taxa with significant differences among eggs, nymph and adult (Fig. 8b). At the level of genus, the biomarker showed the significant differences among the eggs, nymph and adult stages. The genera Blattabacterium and Lactobacillus were significant different genera between the eggs stage and the other two life stages. Butyricimonas, Parabacteroides, Rikenellaceae, Christensenellaceae, Ruminococcaceae, and Desulfovibrio were significant different genera between the nymph stage and the other two life stages. Moreover, the genera Bacteroides, Dysgonomonas, Porphyromonadaceae and Alistipes were the between the adult stage and the other two life stages.

The functional gene composition in each life stage was inferred by comparing $16 \mathrm{~S}$ sequencing data via PICRUSt software, and the differences and changes of metabolic pathways of functional genes in gut microbiome among the samples of different life stages could be observed by analysis of KyotoEncyclopedia of Genes and Genomes (KEGG) metabolic pathways. The representative abundance of functional genes in the metabolic pathways were displayed in Fig. 9, and the results showed the top 3 
representative abundance of functional genes in the metabolic pathways in the eggs, nymph and adult life stages were Global and overview maps, Amino acid metabolism and Carbohydrate metabolism. Moreover, a total of 6 functional genes in the metabolic pathways showed significant differences between the eggs stage and the other life stages $(p<0.05)$.

\section{Discussion}

With the development of next-generation sequencing (NGS) technology, bacteria gene sequencing has become more convenient (Macas et al 2011; Aloisio et al 2016). In this study, we used 16S rRNA gene Illumina HiSeq sequencing to study the gut microbiome in eggs, nymph and adult life stages of Periplaneta americana. The results from Alpha-diversity analysis showed that the richness and diversity of gut microbiome in eggs stage was significant lower than that in nymph and adult stages. However, the numbers of the OTUs shared by eggs, nymph and adult stages were high, and the predominant bacteria in the three life stages were similar. The results suggested that the gut microbiome of Periplaneta americana might be inherited to the offspring from their parents.

Microbial communities are particularly prominent in the digestive tract of most insects and animals (Rowland et al 2017). Like other animals, the insect gut microbiome is an important factor which may be related to many physiological functions of their host such as food digestion, tolerance, development and immunity, etc. (Chen et al 2016; Ceja-Navarro et al 2015; Aksoy 2018). The histogram of relative phylum abundance showed that regardless of the Periplaneta americana in eggs, nymph or adult stages, Bacteroidetes, Firmicutes and Proteobacteria were the predominant bacteria. Many previous studies have shown that both Bacteroidetes and Firmicutes are associated with the nutrient absorption of the host such as digestion of the carbohydrates that synthesize short chain fatty acids (Macfarlane and Macfarlane 2003; Liu et al 2017). Recent studies suggested that Bacteroidetes seem to establish a close interaction with their host early in lifetime and played an important role in the immunomodulation of their host (Ivanov et al 2008; Gibiino et al 2018). In addition, Firmicutes can also inhibit the invasion of opportunistic pathogens and prevent intestinal inflammation in vivo (Atarashi et al 2011; Zhang et al 2015). The increment of Bacteroidetes and Firmicutes abundance gut microbiome of eggs stage would likely concomitantly increase the digestive and immune physiological ability. Furthermore, the comparison of the structure of gut microbiome showed significant differences between eggs stage and the other two life stages, which suggesting that Bacteroidetes and Firmicutes may have close related to the physiology of Periplaneta americana, especially in eggs stage.

The results of LefSe analysis showed the significant differences in gut microbiome among eggs, nymph and adult stages on the genus level. The biomarker demonstrated the significant different genera between eggs stage and other two stages constitute Blattabacterium and Lactobacillus. Blattabacterium is a genus of obligate mutualistic endosymbiont bacteria that are believed to inhabit all species of cockroach studied to date, with the exception of the genus Nocticola (Lo et al 2007). It serves a vital role in nitrogen cycling, which is important to their host for using such as woods and other raw materials to synthesize all of the essential amino acids and several vitamins (Sabree et al 2009; Patiño-Navarrete et al 
2014). Moreover, Blattabacterium seems to be inherited to succeeding generations of the host by infection of the mother's eggs prior to their fertilization (Carrasco et al 2014), which indicating the gut microbiome of Periplaneta americana with ability of heredity. Besides, Lactobacillus is a probiotic which can protect the host against potential invasions by pathogens and other diseases (Martin et al 2013; Ford et al 2014). Therefore, Blattabacterium and Lactobacillus constitutes a significant component of the gut microbiome in eggs stage of Periplaneta americana, which indicating that they may play an important role in the nutrient absorption and disease resistance of Periplaneta americana, especially in eggs life stages.

The KEGG analysis revealed the distribution of functional genes in the metabolic pathways in eggs, nymph and adult stages of Periplaneta americana. The abundance of functional genes in the metabolic pathways of Translation, Nucleotide metabolism, and Cell growth and death significant increased in eggs stage, which indicating presence of gut microbiome may influence the growth and development of the host (Sommer et al 2013; Coon et al 2014). In addition, with the growth and development of Periplaneta americana, the abundance of functional genes in the metabolic pathways of Membrane transport, Signal transduction and Xenobiotics biodegradation and metabolism significant increased in nymph and adult stage, which indicating the gut microbiome may influence more and more different aspects of physiological and social activities of Periplaneta americana nymphs and adults (Parashar et al 2016; Kohl 2012; Paniagua et al 2018). The highest abundance of functional genes in the metabolic pathways of Global and overview maps, Amino acid metabolism, and Carbohydrate metabolism in eggs, nymph and adult stages of Periplaneta americana, and we believed the gut microbiome may play an important role in the basic physiological functions of Periplaneta americana across its whole life stages.

The structure and composition of gut microbiome in nymph and adult stages of Periplaneta americana exhibited more complex than those in eggs stage, suggesting that there may be many underlying reasons for physiological regulation in nymph and adult stages. Periplaneta americana has strong ability of environmental stress resistance so that it can survive in harsh environment. In order to adapt to the complicated and changeable environment, the gut microbiome may change with the grows and develops of Periplaneta americana (Benson et al 2010; Dehler et al 2017). Furthermore, the compositions of gut microbiome significantly correlated with diet of the host, especially in the elder stage (Claesson et al 2012; Scott et al 2013; Serino et al 2012). Therefore, we believed the complex intestinal bacterial communities of nymph and adult stages might be influenced by the environment and diet, and play a vital role in the physiology of Periplaneta americana, especially the environmental adaptability.

Although accumulating researches have focused on the microbial in the insect gut, to data there have been few reports comparing diversity and structure of gut microbiome associated with successive life stages. This study is the first to discuss the differences in the diversity and structure of gut microbiome across the eggs, nymph and adult life stages of Periplaneta americana. The results in this study reveals the diversity and structure of gut microbiome in eggs, nymph and adult life stages of Periplaneta americana, and suggests the gut microbiome will become more complex and diverse with the grows and develops of Periplaneta americana. However, the gut microbiome of Periplaneta americana may be 
inherited to offspring from their parents, and we believe the gut microbiome in every life stages of Periplaneta americana may play their respective physiological regulating roles in different life stages. However, the mechanisms of how the gut microbiome affects the physiological functions of Periplaneta americana are still unknown, and require further study. This study helps advance our understanding of the physiology and other characteristics of Periplaneta americana, which could promote the development of effective management strategies for Periplaneta americana.

\section{Conclusions}

Periplaneta americana is a widely distributed pest of public health importance found in the tropics and subtropics. It is a serious urban pest due to its ability to act as a carrier and transfer many diseasecausing organisms. This first to study the differences in the diversity and structure of gut microbiome in the eggs, nymph and adult life stages of Periplaneta americana systematically. The diversity and structure of gut microbiome in different life stages of Periplaneta americana are significant different, which suggesting the gut microbiome may change with the growth and development of Periplaneta americana. However, the predominant bacteria in the eggs, nymph and adult are similar, indicating the gut microbiome of Periplaneta americana can be inherited to the offspring from their parents across its whole life stages. All representative functional genes in metabolic pathways are both related to various physiological activities of eggs, nymph and adult stages, indicating the gut microbiome may influence many aspects of Periplaneta americana physiological functions such as metabolism, immunity, growth, development, and behavior, etc. In conclusion, this study has revealed differences in diversity and structure of gut microbiome in different life stages of Periplaneta americana, which may help us to better understand its physiological activities and lead to more effective management of cockroaches.

\section{Declarations}

Acknowledgements

This work was funded by the Public Welfare Research and Capacity Building Project of Guangdong Province (No. 2016A030303059 and No. 2017A020211008). The Key Projects of Basic Research and Applied Basic Research of Guangdong Province Normal University (No. 2018KZDXM041).

Author's Contributions

X.B.J. and Z.Y.C. conceived and designed the experiments. Z.Y.C., P.Y.O., B.Y. and L.Y.L. performed the experiments and analyzed the data. X.B.J. and Z.Y.C. wrote the paper. All authors read and approved the manuscript.

Compliance with ethical standards

Conflict of interest 
The authors declare no competing financial interests.

Ethical approval

The article does not contain any studies with human participants or animals performed by any of the authors.

\section{References}

Aksoy S (2018) Insect Gut Microbiota: Accessories to the Bite. Cell Host Microbe 23: 8-9. https://doi.org/10.1016/j.chom.2017.12.016

Aloisio I, Quagliariello A, Fanti DS, Luiselli D, Carlotta CD, Albanese D, Corvaglia LT, Faldella G, Gioia D (2016) Evaluation of the effects of intrapartum antibiotic prophylaxis on newborn intestinal microbiota using a sequencing approach targeted to multi hypervariable 16S rDNA regions. Appl Microbiol Biotechnol 100: 5537-5546. https://doi.org/10.1007/s00253-016-7410-2.

Atarashi K, Tanoue T, Shima T, Imaoka A, Kuwahara T, Momose Y, Cheng G, Yamasaki S, Saito T, Ohba Y, Taniquchi T, Takeda K, Hori S, Ivanov II, Umesaki Y, Itoh K, Honda K (2011) Induction of colonic regulatory T cells by indigenous Clostridium species. Science 331: 337-341.

https://doi.org/10.1126/science.1198469

Ayako W, Ludek Z, Godfrey N, Roel ofs WL, Zhang A, Schal C (2015) Gut bacteria mediate aggregation in the German cockroach. Proc Natl Acad Sci U. S. A 112: 15678-15683.

https://doi.org/10.1073/pnas.1504031112

Bäckhed F, Ley RE, Sonnenburg JL, Perterson DA, Gordon JI (2005) Host-bacterial mutualism in the human intestine. Science 307: 1915-1920. https://doi.org/10.1126/science.1104816

Baggio-Deibler MV, Da CFM, Monteiro AC, Souza-Pollo A, Franco Lemos MV (2018) Management of the American cockroach's oothecae: The potential of entomopathogenic fungi control. J Invertebr Pathol 153: 30-34. https://doi.org/10.1016/j.jip.2018.02.008

Bell WJ, Adiyodi KG (1981) The American cockroach. Springer Netherlands Publishers, Berlin, pp 1- -14

Benson AK, Kelly SA, Legge R, Ma F, Low SJ, Kim J, Zhang M, Oh PL, Nehrenberg D, Hua K, Kachman SD, Moriyama EN, Walter J, Peterson DA, Pomp D (2010) Individuality in gut microbiota composition is a complex polygenic trait shaped by multiple environmental and host genetic factors. Proc Natl Acad Sci USA 7: 18933-18938. https://doi.org/10.1073/pnas.1007028107

Bokulich NA, Subramanian S, Faith JJ, Gevers D, Gordon JI, Knight R, Mills DA, Caporaso JG (2013) Quality-filtering vastly improves diversity estimates from Illumina amplicon sequencing. Nat Methods 10:57-59. https://doi.org/10.1038/nmeth.2276 
Caporase JG, Kuczynski J, Stombaugh J, Bittinger K, Bushman FD, Costello EK, Fierer N, Pena AG, Goodrich JK, Gordon JI (2010) QIIME allows analysis of high-throughput community sequencing data. Nat Methods 7: 335-336. https://doi.org/10.1038/nmeth.f.303

Carrasco P, Pérez-Cobas AE, Van de PC, Baixeras J, Moya A, Latorre A (2014) Succession of the gut microbiota in the cockroach Blattella germanica. Int Microbiol 17:99-109.

https://doi.org/10.2436/20.1501.01.212

Ceja-Navarro JA, Vega FE, Karaoz U, Hao Z, Jenkins S, Lim HC, Kosina P, Infante F, Northen TR, Brodie EL (2015) Gut microbiota mediate caffeine detoxification in the primary insect pest of coffee. Nat Commun 6: 7618. https://doi.org/10.1038/ncomms8618

Chen BS, Teh BS, Sun C, Hu S, Lu XM, Boland W, Shao YQ (2016) Biodiversity and Activity of the Gut Microbiota across the Life History of the Insect Herbivore Spodoptera littoralis. Sci Rep 6: 29505. https://doi.org/10.1038/srep29505

Claesson MJ, Jeffery IB, Conde S, Power SE, O'Connor EM, Cusack S, Harris HM, Coakley M, Lakshminarayanan B, O'Sullivan O, Fitzgerald GF, Deane J, O'Connor M, Harnedy N, O'Connor K, O'Mahony D, van Sinderen D, Wallace M, Brennan L, Stanton C, Marchesi JR, Fitzgerald AP, Shanahan F, Hill C, Ross RP, O'Toole PW (2012) Gut microbiota composition correlates with diet and health in the elderly. Nature 488: 178-184. https://doi.org/10.1038/nature11319

Coon KL, Vogel KJ, Brown MR, Strand MR (2014) Mosquitoes rely on their gut microbiota for development. Mol Ecol 23: 2727-2739. https://doi.org/10.1038/nature11319

Crotti E, Balloi A, Hamdi C, Sansonno L, Marzorati M, Gonella E, Favia G, Cherif A, Bandi C, Alma A, Daffonchio D (2012) Microbial symbionts: a resource for the management of insect-related problems. Microb Biotechnol 5: 307-317. https://doi.org/10.1111/j.1751-7915.2011.00312.x

Dehler CE, Secombes CJ, Martin SAM (2017) Environmental and physiological factors shape the gut microbiota of Atlantic salmon parr (Salmo salar L.). Aquaculture 467: 149-157.

https://doi.org/10.1016/j.aquaculture.2016.07.017

Douglas AE (2014) The Molecular Basis of Bacterial-Insect Symbiosis. J Mol Biol 426: 3830-3837. https://doi.org/10.1016/j.jmb.2014.04.005

Edgar RC (2010) Search and clustering orders of magnitude faster than BLAST. Bioinformatics 26: 24602461. https://doi.org/10.1093/bioinformatics/btq461

Engel P, Moran NA (2013a) The gut microbiota of insects - diversity in structure and function. FEMS Microbiol Rev 37: 699-735. https://doi.org/10.1111/1574-6976.12025

Engel P, Moran NA (2013b) Functional and evolutionary insights into the simple yet specific gut microbiota of the honey bee from metagenomic analysis. Gut Microbes 4: 60-65. 
Ford AC, Quigley EM, Lacy BE, Lembo AJ, Saito YA, Schiller LR, Soffer EE, Spiegel BM, Moayyedi P (2014) Efficacy of prebiotics, probiotics, and synbiotics in irritable bowel syndrome and chronic idiopathic constipation: systematic review and meta-analysis. Am J Gastroenterol 109:1547-61. https://doi.org/10.1038/ajg.2014.202

Gibiino G, Lopetuso LR, Scaldaferri F, Rizzatti G, Binda C, Gasbarrini A (2018) Exploring Bacteroidetes: Metabolic key points and immunological tricks of our gut commensals. Digest Liver Dis 7: 635- -639. https://doi.org/10.1016/j.dld.2018.03.016

Ivanov II, Frutos Rde L, Manel N, Yoshinaga K, Rifkin DB, Sartor RB, Finlay BB, Littman DR (2008) Specific Microbiota Direct the Differentiation of IL-17-Producing T-Helper Cells in the Mucosa of the Small Intestine. Cell Host \& Microbe 4: 337-349. https://doi.org/10.1016/j.chom.2008.09.009

Kaltenpoth M, Engl T, Clay K (2014) Defensive microbial symbionts in Hymenoptera. Functional Ecology 28: 315-327. https://doi.org/10.1111/1365-2435.12089

Kohl KD (2012) Diversity and function of the avian gut microbiota. J Comp Physiol B 182:591-602. https://doi.org/10.1007/s00360-012-0645-z

Kwong WK, Mancenido AL, Moran NA (2017) Immune system stimulation by the native gut microbiota of honey bees. R Soc Open Sci 4: 170003. https://doi.org/10.1098/rsos.170003

Li S, Zhu S, Jia Q, Yuan D, Ren C, Li K, Liu S, Cui Y, Zhao H, Cao Y, Fang G, Li D, Zhao X, Zhang J, Yue Q, Fan Y, Yu X, Feng Q, Zhan S (2018) The genomic and functional landscapes of developmental plasticity in the American cockroach. Nat Commun 9: 1008. https://doi.org/10.1038/s41467-018-03281-1

Liu JQ, Wang YH, Fang XT, Xie M, Zhang YS, Hou SS, Chen H, Chen GH, Zhang CL (2017) Dietary energy level affects the composition of cecal microbiota of starter Pekin ducklings. Czech J Anim Sci 63: 24-31. https://doi.org/10.5713/ajas.18.0683

Lo N, Beninati T, Stone F, Walker J, Sacchi L (2007) Cockroaches that lack Blattabacterium endosymbionts: the phylogenetically divergent genus Nocticola. Bio Lett 3: 327-330. https://doi.org/10.1098/rsbl.2006.0614

Macas J, Kejnovsky E, Neumann P, Novák P, Kobližková A, Vyskot B (2011) Next Generation SequencingBased Analysis of Repetitive DNA in the Model Dioceous Plant Silene latifolia. PLoS ONE 6: e27335. https://doi.org/10.1371/journal.pone.0027335

Macfarlane S, Macfarlane GT (2003) Regulation of short-chain fatty acid production. Proc Nutr Soc 62: 67-72. https://doi.org/10.1079/pns2002207 
Magoč T, Salzberg SL (2011) FLASH: fast length adjustment of short reads to improve genome assemblies. Bioinformatics 27: 2957-2963. https://doi.org/10.1093/bioinformatics/btr507

Martin R, Miquel S, Ulmer J, Kechaou N, Langella P, Bermúdez-Humarán LG (2013) Role of commensal and probiotic bacteria in human health: a focus on inflammatory bowel disease. Microb Cell Fact 12: 71. https://doi.org/10.1186/1475-2859-12-71

Mullins DE (2015) Physiology of environmental adaptations and resource acquisition in cockroaches. Annu Rev Entomol 60: 473-492. https://doi.org/10.1146/annurev-ento-011613-162036

Nasirian H (2018) Infestation of cockroaches (Insecta: Blattaria) in the human dwelling environments: A systematic review and meta-analysis. Acta Trop 167: 86-98.

https://doi.org/10.1016/j.actatropica.2016.12.019

Pai HH (2015) Multidrug resistant bacteria isolated from cockroaches in long-term care facilities and nursing homes. Acta Trop 125: 18-22. https://doi.org/10.1016/j.actatropica.2012.08.016.

Paniagua Voirol LR, Frago E, Kaltenpoth M, Hilker M, Fatouros NE (2018) Bacterial Symbionts in Lepidoptera: Their Diversity, Transmission, and Impact on the Host. Front Microbiol 9: 556. https://doi.org/10.3389/fmicb.2018.00556

Parashar A, Udayabanu M (2016) Gut microbiota regulates key modulators of social behavior. Eur Neuropsychopharmacol 26:78-91. https://doi.org/10.1016/j.euroneuro.2015.11.002

Patiño-Navarrete R, Piulachs MD, Belles X, Moya A, Latorre A, Peretó J (2014) The cockroach Blattella germanica obtains nitrogen from uric acid through a metabolic pathway shared with its bacterial endosymbiont. Biol Lett 10: 20140407. https://doi.org/10.1098/rsbl.2014.0407

Rowland I, Gibson G, Heinken A, Scott K, Swann J, Thiele I, Tuohy K (2017) Gut microbiota functions: metabolism of nutrients and other food components. Eur J Nutr 57: 1-24.

https://doi.org/10.1007/s00394-017-1445-8

Sabree ZL, Kambhampati S, Moran NA (2009) Nitrogen recycling and nutritional provisioning by Blattabacterium, the cockroach endosymbiont. Proc Natl Acad Sci USA 106: 19521-19526. https://doi.org/10.1073/pnas.0907504106

Scott KP, Gratz SW, Sheridan PO, Flint HJ, Duncan SH (2012) The influence of diet on the gut microbiota. Pharmacol Res 69: 52-60. https://doi.org/10.1016/j.phrs.2012.10.020

Segata N, Izard J, Waldron L, Gevers D, Miropolsky L, Garrett WS, Huttenhower C (2011) Metagenomic biomarker discovery and explanation. Genome Biol 12: R60. https://doi.org/10.1186/gb-2011-12 6-r60

Serino M, Luche E, Gres S, Baylac A, Bergé M, Cenac C, Waget A, Klopp P, lacovoni J, Klopp C, Mariette J, Bouchez O, Lluch J, Ouarné F, Monsan P, Valet P, Roques C, Amar J, Bouloumié A, Théodorou V, Burcelin R 
(2012) Metabolic adaptation to a high-fat diet is associated with a change in the gut microbiota. Gut 61: 543-553. https://doi.org/10.1136/gutjnl-2011-301012

Shin SC, Kim SH, You H, Kim B, Kim AC, Lee KA, Yoon JH, Ryu JH, Lee WJ (2011) Drosophila microbiome modulates host developmental and metabolic homeostasis via insulin signaling. Science 334: 670-674. https://doi.org/10.1126/science.1212782

Sommer F, Backhed F (2013) The gut microbiota-masters of host development and physiology. Nat Rev Microbiol 11: 227-238. https://doi.org/10.1038/nrmicro2974

Tinker KA, Ottesen EA (2016) The core gut microbiome of the American cockroach, Periplaneta americana, is stable and resilient to dietary shifts. Appl Environ Microbiol 82: 6603-6610. https://doi.org/10.1128/AEM.01837-16

Walters W, Hyde ER, Berg-Lyons D, Ackermann G, Humphrey G, Parada A, Gilbert JA, Jansson JK, Caporaso JG, Fuhrman JA, Apprill A, Knight R (2016) Improved Bacterial 16S rRNA Gene (V4 and V4-5) and Fungal Internal Transcribed Spacer Marker Gene Primers for Microbial Community Surveys. Msystems 1: e00009-00015. https://doi.org/10.1128/mSystems.00009-15

Yu Y, Lee C, Kim J, Hwang S (2005) Group-specific primer and probe sets to detect methanogenic communities using quantitative real-time polymerase chain reaction. Biotechnol Bioeng 89: 670- -679. https://doi.org/10.1002/bit.20347

Yun J, Hwang JS,

Lee DG (2017) The antifungal activity of the peptide, periplanetasin-2, derived from American cockroach Periplaneta americana. Biochem J 474: 3027-3043. https://doi.org/10.1042/bcj20170461

Zhang J, Guo Z, Xue Z, Sun Z, Zhang M, Wang L, Wang G, Wang F, Xu J, Cao H, Xu H, Lv Q, Zhong Z, Chen Y, Qimuge S, Menghe B, Zheng Y, Zhao L, Chen W, Zhang H (2015) A phylo-functional core of gut microbiota in healthy young Chinese cohorts across lifestyles, geography and ethnicities. ISME J 9: 19791990. https://doi.org10.1038/ismej.2015.11

\section{Figures}




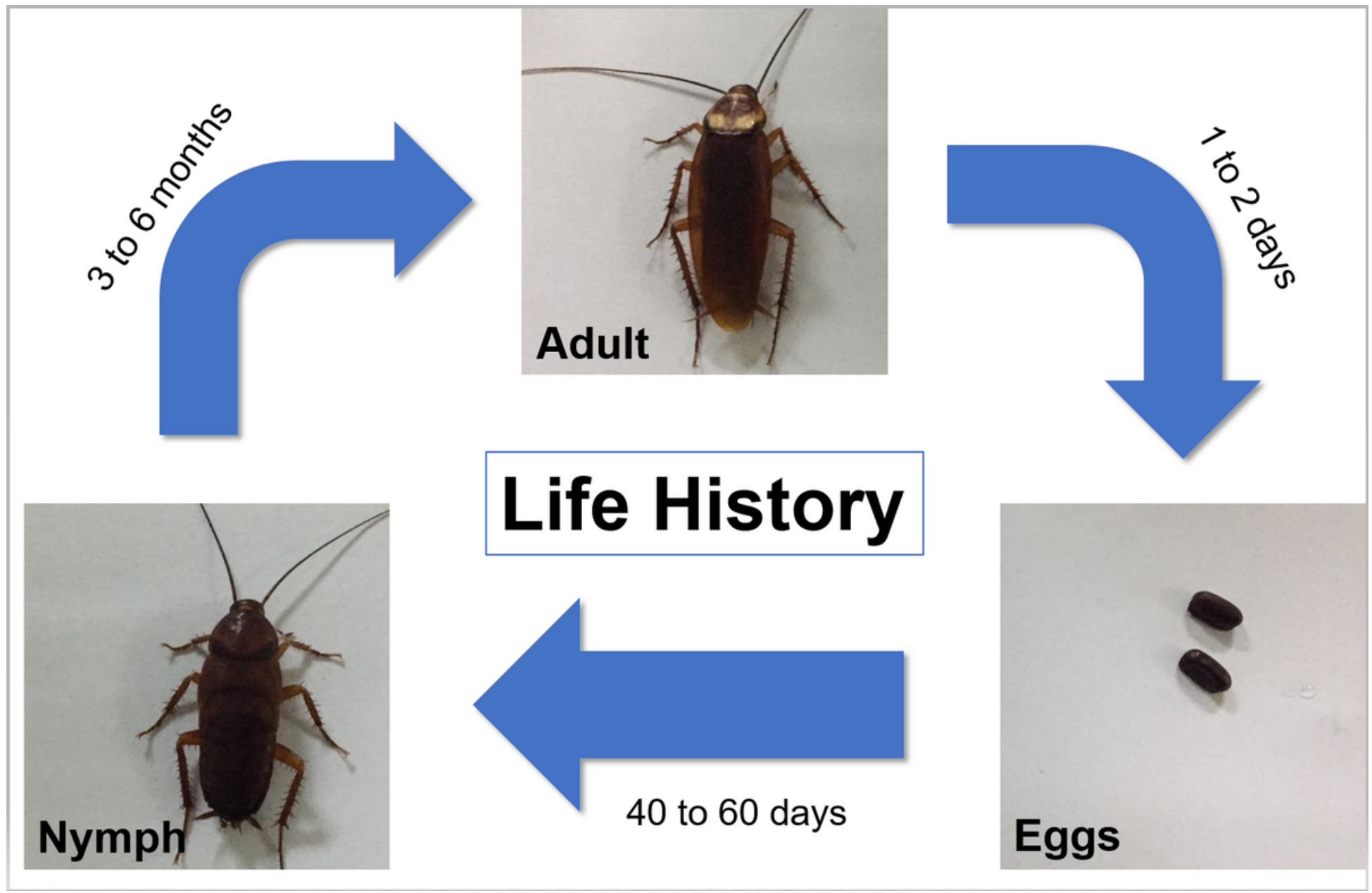

Figure 1

Overview of the development stages of Periplaneta americana.

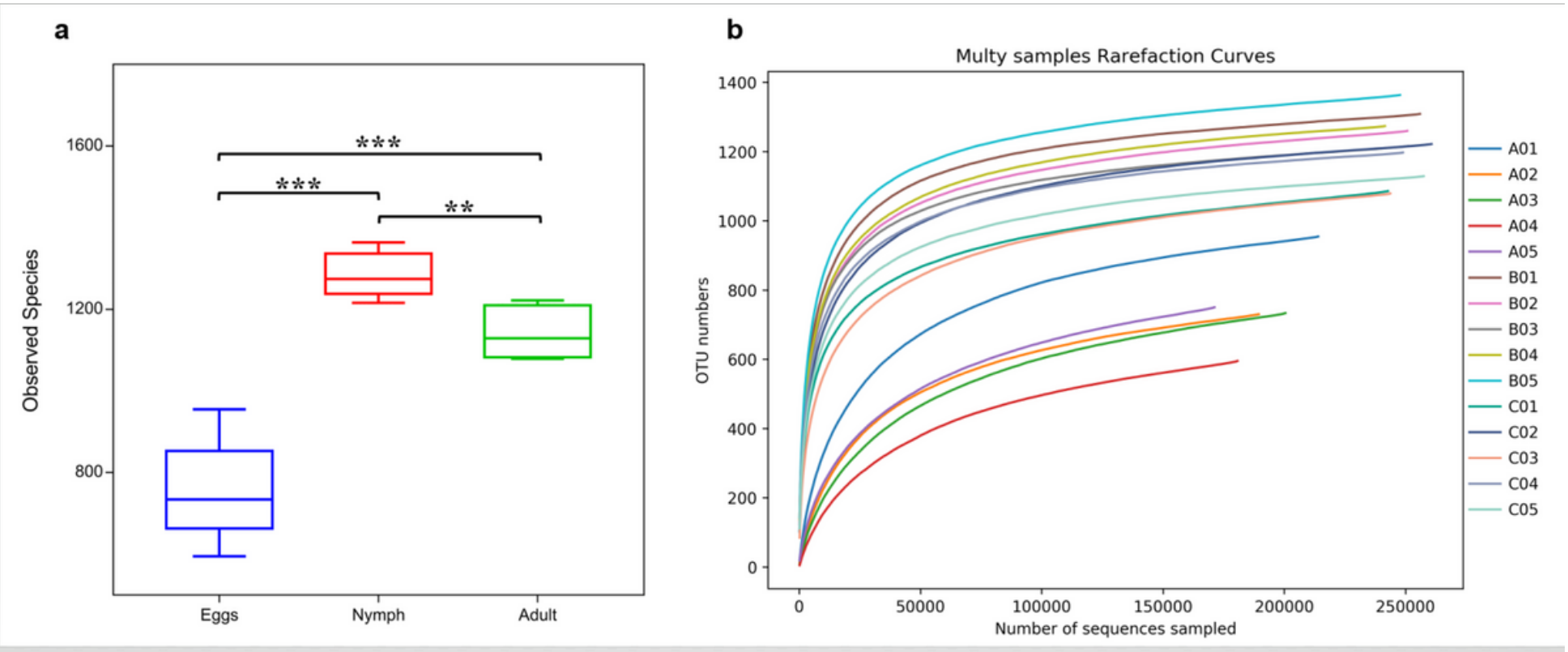

Figure 2 
Boxplot and rarefaction curves of operational taxonomic units (OTUs). (a) Boxplot of OTUs of observed species. The $x$-axis shows the observed species (OTUs) and the y-axis shows different groups. The OTU similarity threshold of $97 \%$ was considered. Boxes represent the interquartile range (IQR) between the 25th and 75th peroentile, and the horizontal line inside the box defines the median. Whiskers represent the maximum and minimum values within 1.5 times the IQR from the 25th and 75th peroentile. ${ }^{* \star} p<0.01$, $\star \star \star * \quad p<005$ (Student's t-test). (b) The rarefaction curves of OTUs. The $x$-axis shows the number of valid sequences per sample and the $y$-axis shows the observed species (OTUs). Each curve in the graph is shown in a different color and represents a different sample. The curves were precipitous at the beginning, which indicated that the number of OTUs increased rapidly as the sequencing depth increased. Eventually the curves tended to plateau which indicated that the number of OTUs detected was decreased as the number of extracted sequences increased.
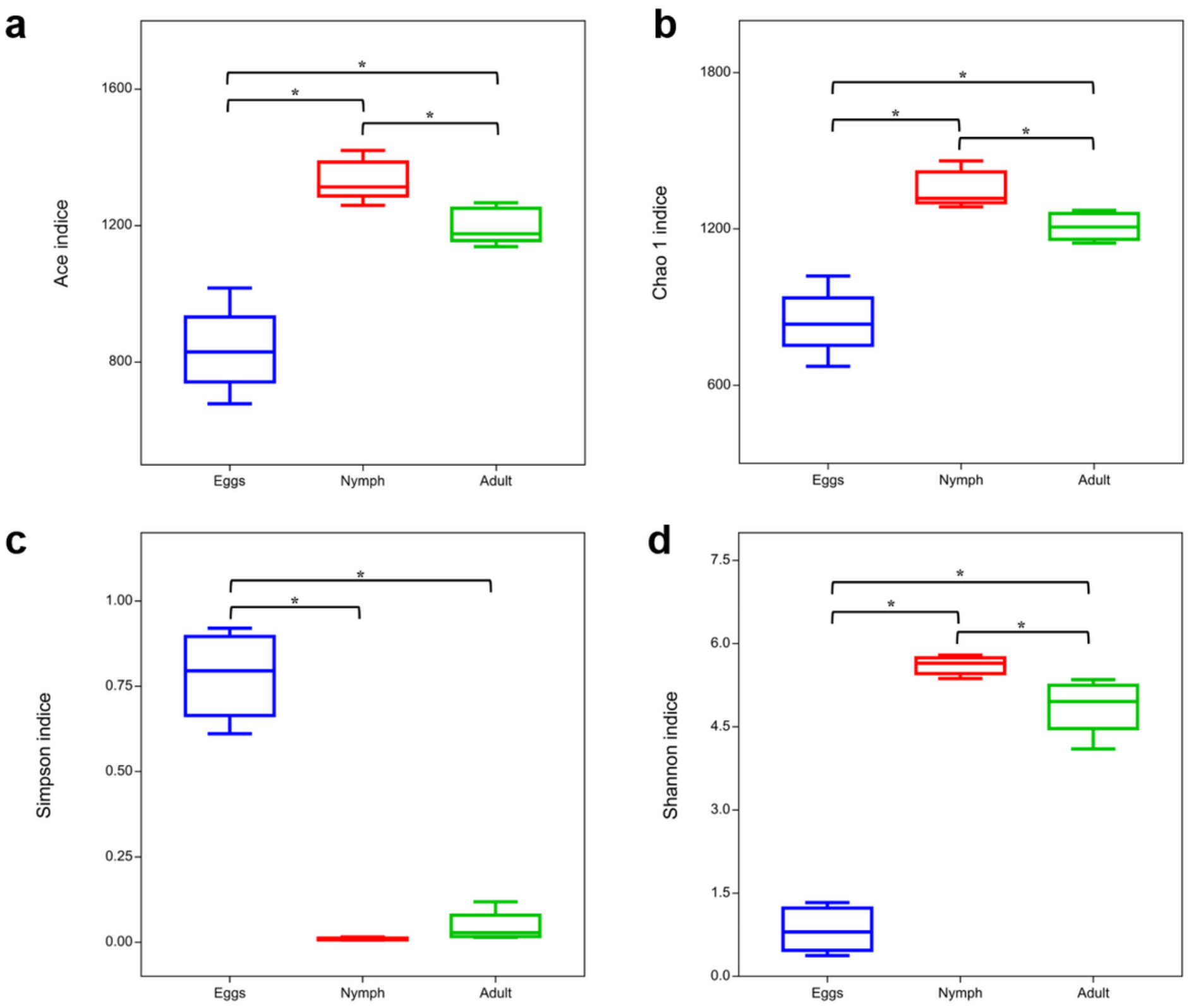

Figure 3 
Boxplot of Alpha-diversity indices. The (a) Ace and (b) Chao 1 indices reflect the OTU abundance in samples. (c) Shannon and (d) Simpson indices reflect the diversity of OTU in samples. The greater the Ace or Chao 1 indices, the higher the expected species richness of the microbiome; the smaller the Simpson or the greater the Shannon indices, the higher the diversity of the microbiome. Boxes represent the interquartile range (IQR) between the 25th and 75th peroentile, and the horizontal line inside the box defines the median. Whiskers represent the maximum and minimum values within 1.5 times the IQR from the 25th and 75th peroentile. ${ }^{*} p<0.05$ (Student's t-test).

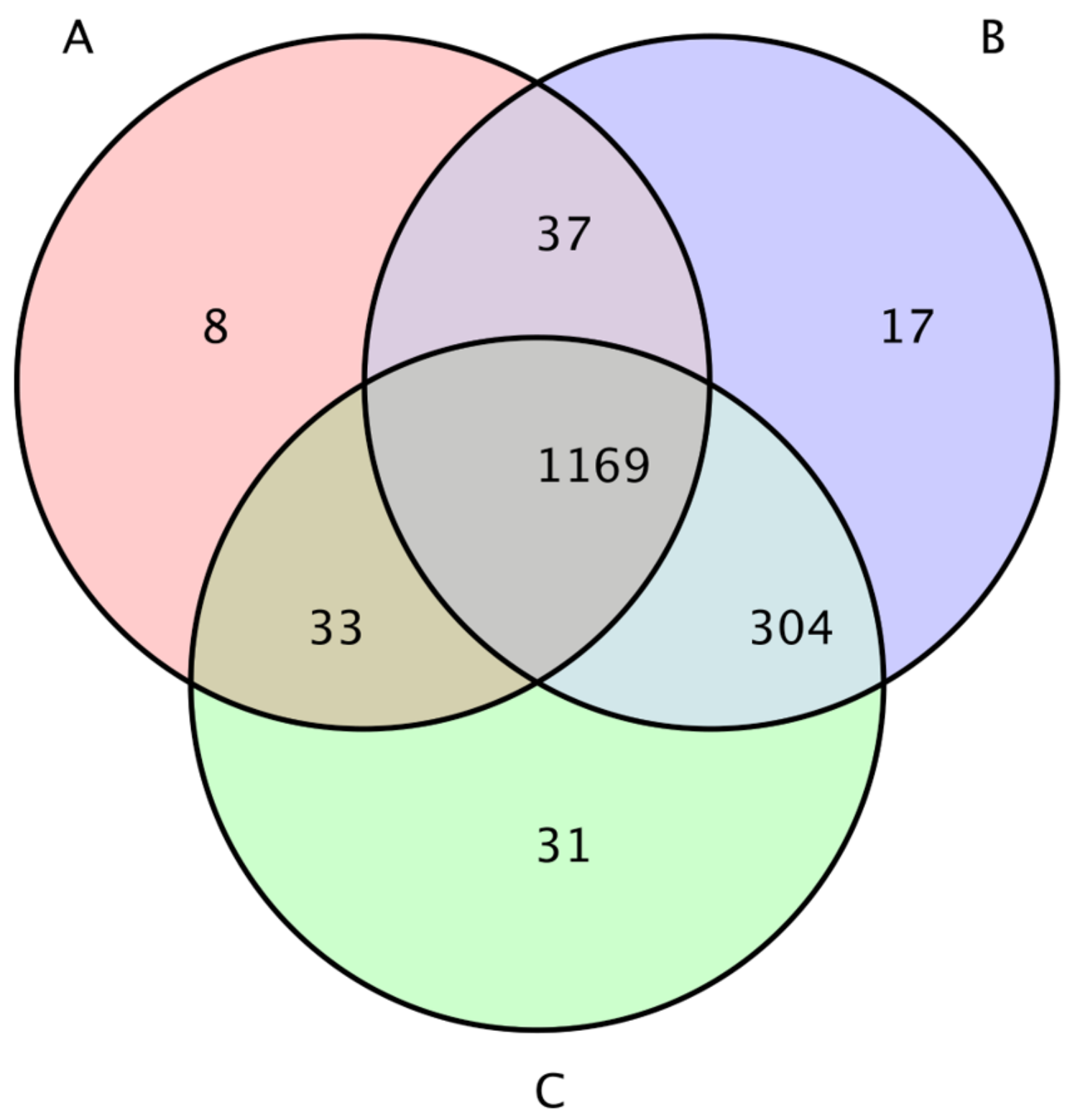

Figure 4 
Venn diagram of eggs, nymph and adult gut samples. The numbers represent the number of unique and shared OTUs in the gut samples at different life stage of Periplaneta americana. A refers to the gut samples from the eggs of Periplaneta americana. B refers to the gut samples from the nymph of Periplaneta americana. $\mathrm{C}$ refers to the gut samples from the adult of Periplaneta americana. Each life stage included five biological replicates.

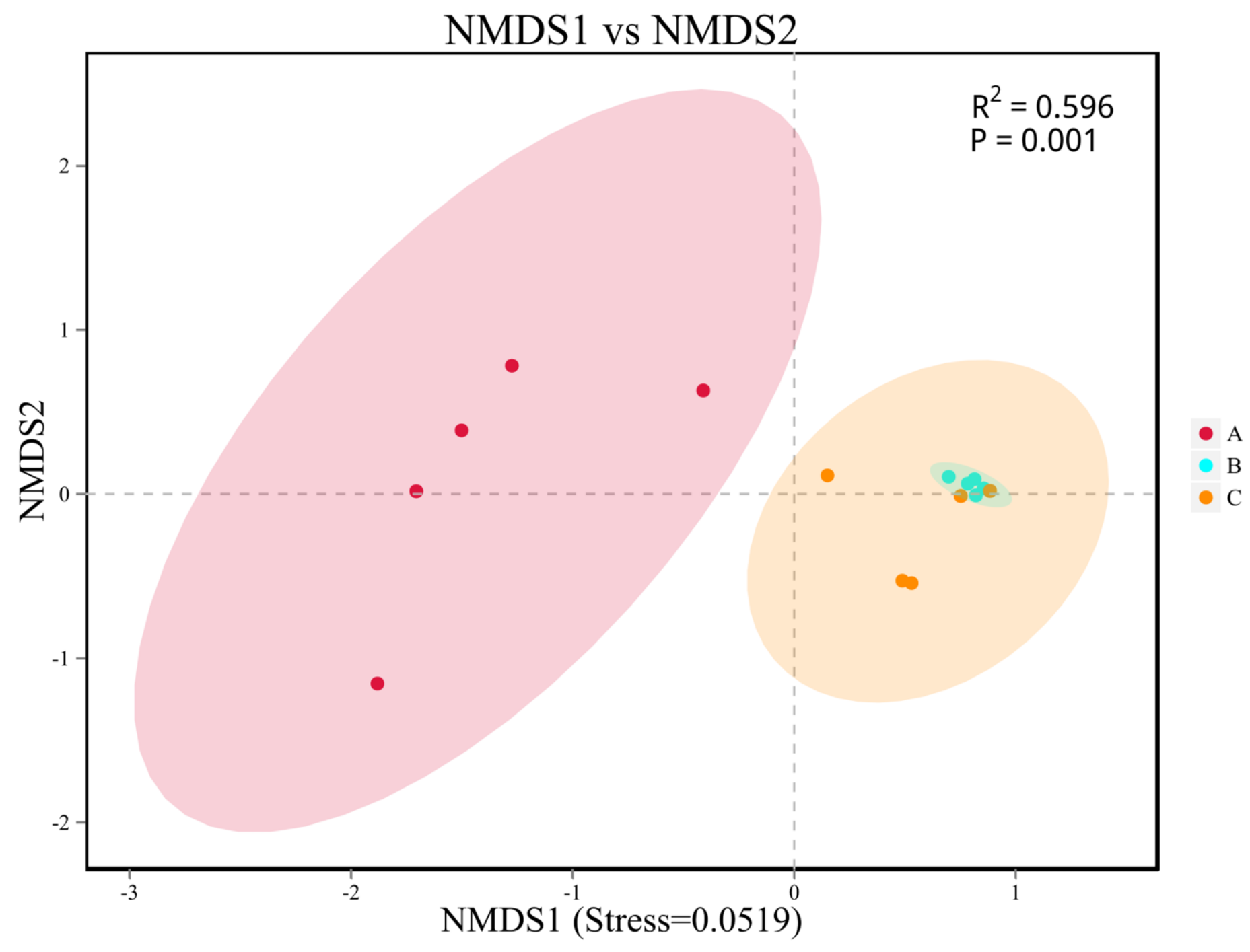

\section{Figure 5}

Nonmetric multidimensional scaling (NMDS) analysis. Each point in the graph represents one sample and each color represents different groups. A refers to the gut samples from the eggs of Periplaneta americana. $B$ refers to the gut samples from the nymph of Periplaneta americana. $C$ refers to the gut samples from the adult of Periplaneta americana. The distances were determined using unweighted Unifrac method with OTUs. The closer of the distance between points the samples in the graph, the higher their similarity. Stress lower than 0.2 indicates that the NMDS analysis is reliable. 

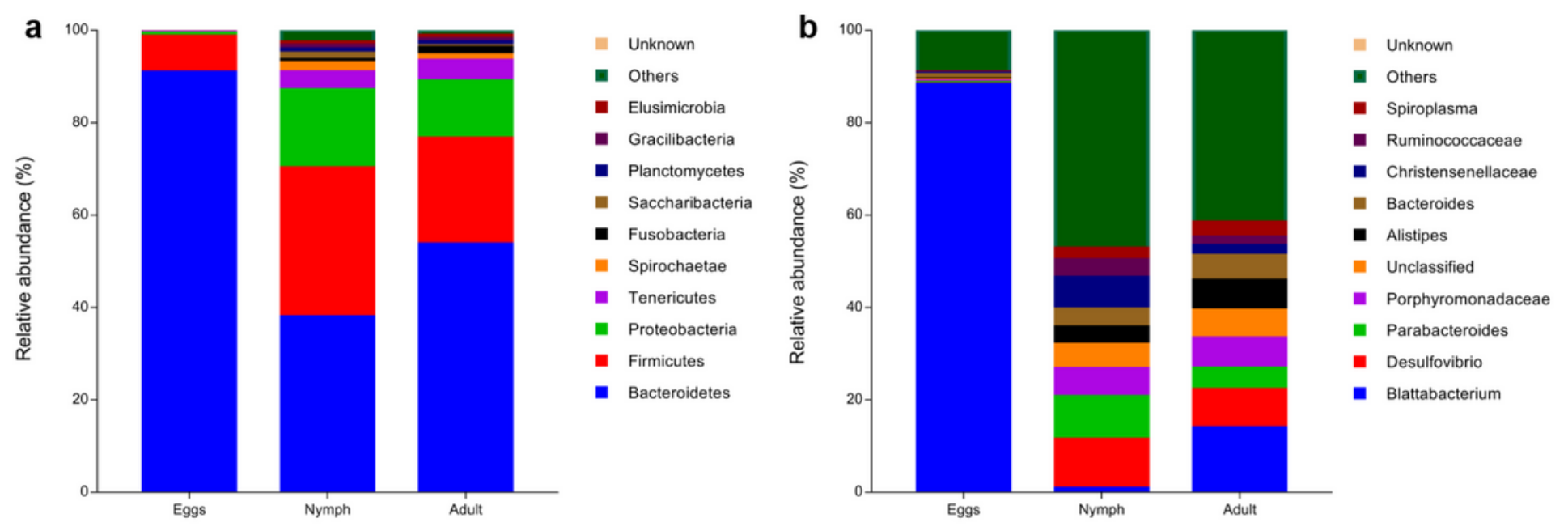

\section{Figure 6}

Relative abundance of bacteria at the phylum and genus levels in eggs, nymph and adult samples of Periplaneta americana. The $x$-axis represents groups and the $y$-axis represents relative abundance presented as percentage. (a) Relative abundance of the top 10 bacteria at the level of phylum. (b) Relative abundance of the top 10 bacteria at the level of genus. Only the top 10 bacteria are shown in the graph, other species are combined as "Other". Each life stages included five biological replicates.

a

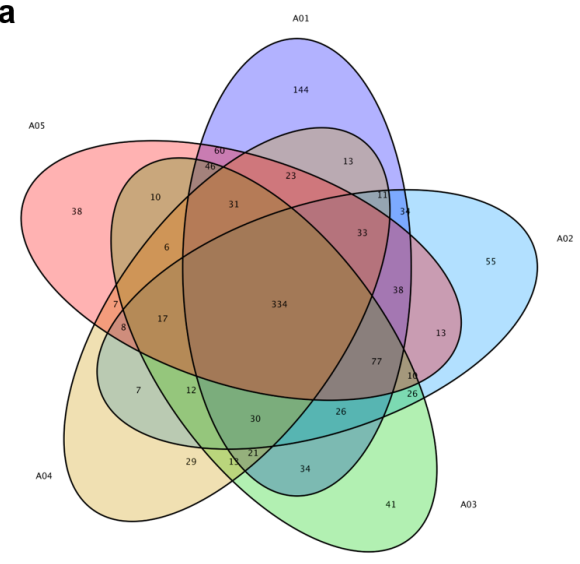

b

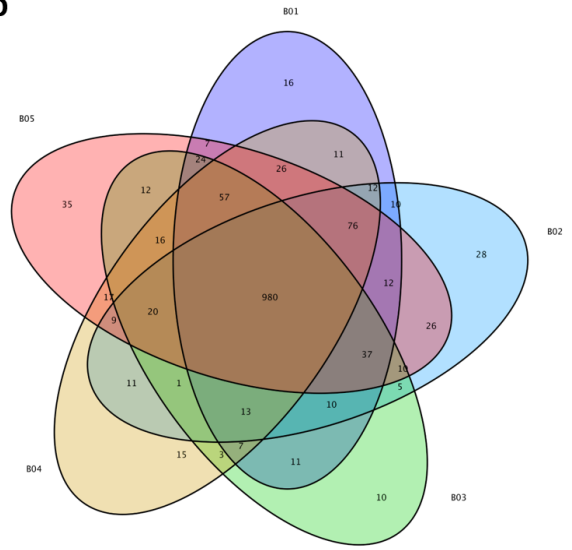

C

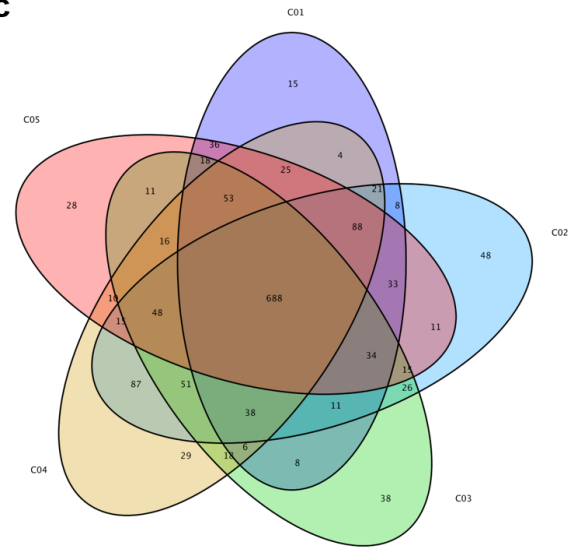

Figure 7

Venn diagram of Periplaneta americana eggs, nymph and adult samples. The diagrams represent the numbers of the shared OTUs (at $97 \%$ sequence identify) in eggs, nymph and adult samples individuals depending on overlaps. (a) The number of OTUs shared by Periplaneta americana eggs samples. (b) The number of OTUs shared by Periplaneta americana nymph samples. (c) The number of OTUs shared by Periplaneta americana adult samples. 


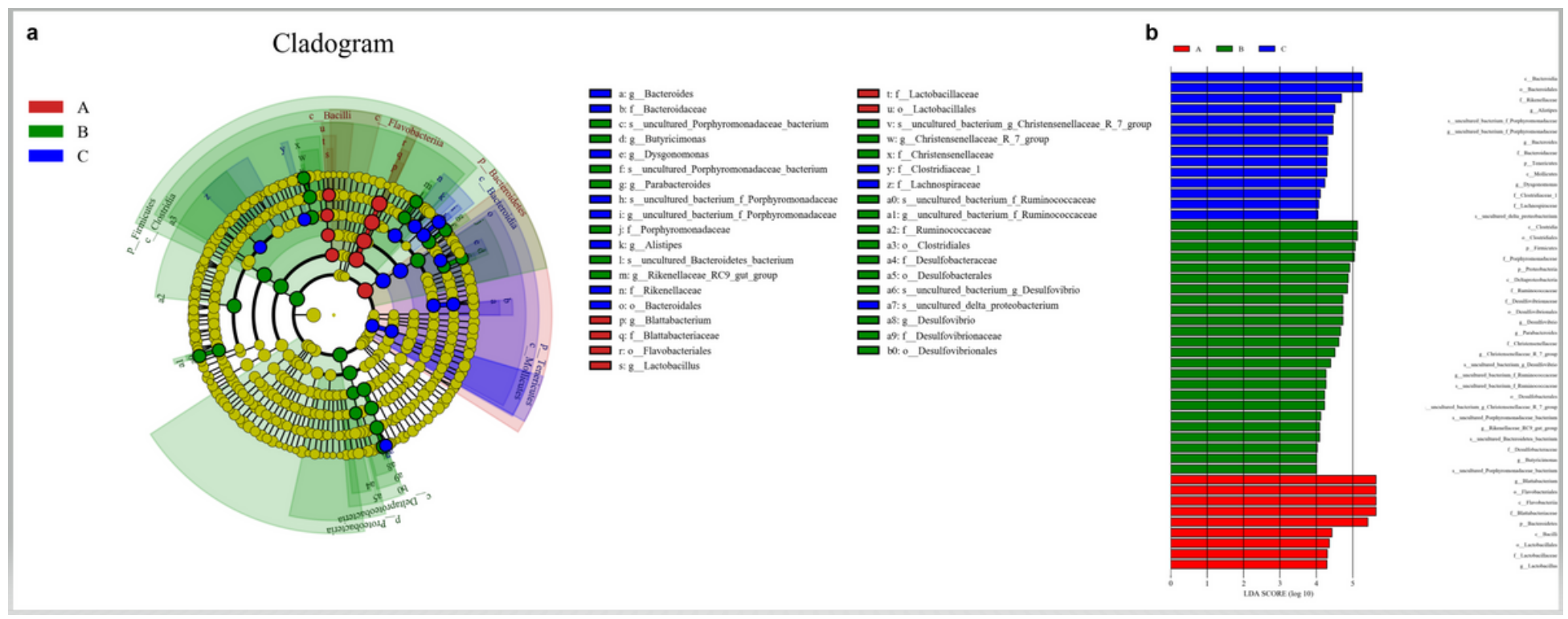

Figure 8

The LefSe analysis of the eggs, nymph and adult samples. (a) The Cladogram shows the microbial species with significant differences in the three life stages. The species classification at the level of phylum, class, order, family, genus and species are shown from the inside to the outside. Red, green and blue refers to eggs, nymph and adult life stages. The red, green and blue nodes in the phylogenetic tree represent microbial species that play an important role in the eggs, nymph and adult life stages. The yellow nodes represent the species with no significant differences among three life stages. (b) While the LDA score greater than the estimated value, the species with significant difference. The length of the histogram represents the LDA score which indicates the degree of influence of species with significant difference between different life stages. The default score is 4.0. 


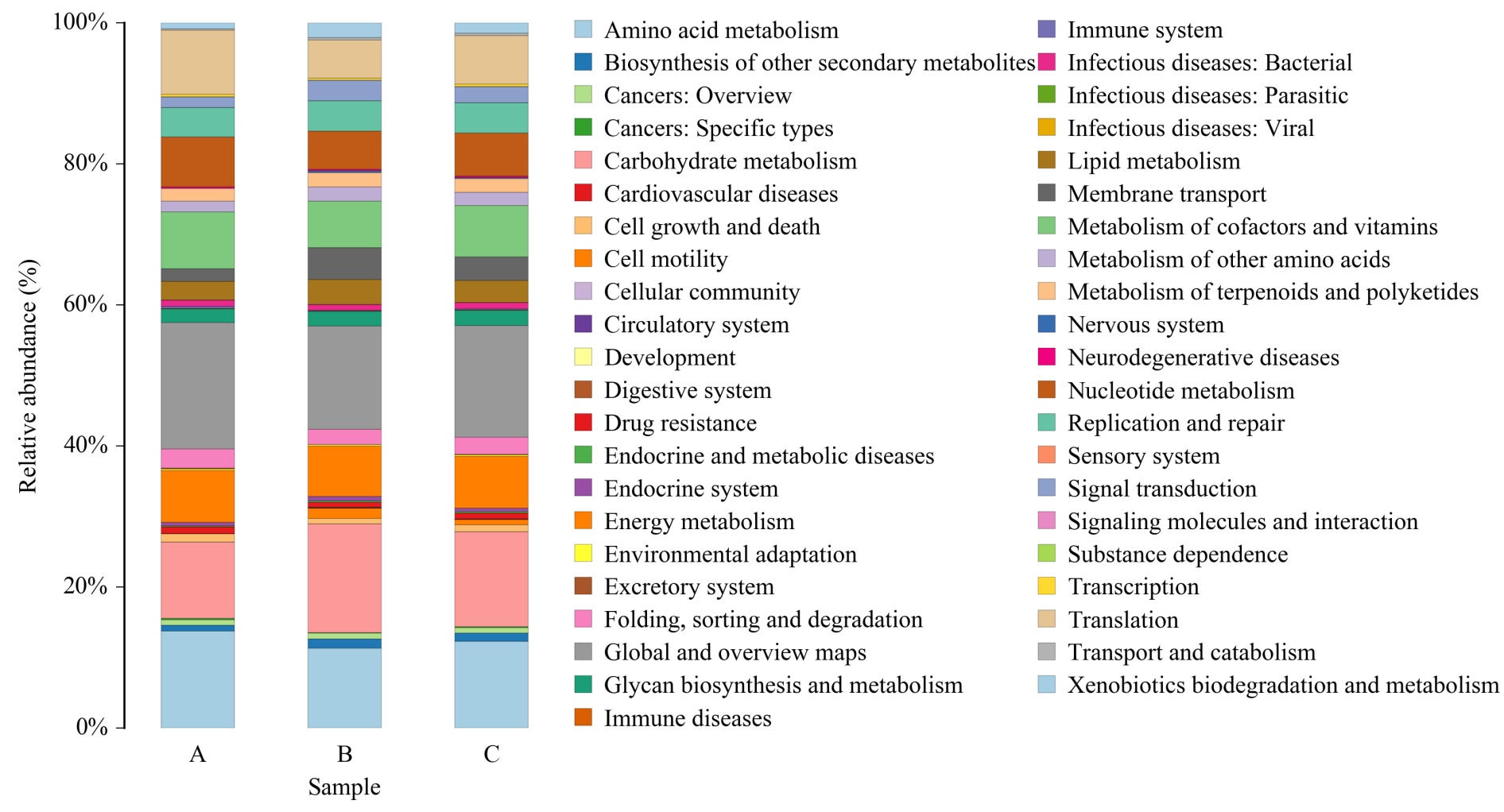

\section{Figure 9}

Relative abundance of metabolic pathways related to functional genes in eggs, nymph and adult samples of Periplaneta americana. The presentative metabolic pathways are shown in the graph. A refers to the gut samples from the eggs of Periplaneta americana. B refers to the gut samples from the nymph of Periplaneta americana. $\mathrm{C}$ refers to the gut samples from the adult of Periplaneta americana. Each life stages included five biological replicates. Different functional genes in metabolic pathways of Periplaneta americana are represented by different color. 